\title{
Reversing the manual digit bias in two-digit number comparison
}

\author{
Thomas J. Faulkenberry \\ Tarleton State University \\ Alexander Cruise \\ Ariel University \\ Samuel Shaki \\ Ariel University
}

\begin{abstract}
Though recent work in numerical cognition has supported a strong tie between numerical and spatial representations (e.g., a mental number line), less is known about such ties in multi-digit number representations. Along this line, Bloechle, Huber, and Moeller (2015) found that pointing positions in two-digit number comparison were biased leftward toward the decade digit. Moreover, this bias was reduced in unit-decade incompatible pairs. In the present study, we tracked computer mouse movements as participants compared two-digit numbers to a fixed standard (55). Similar to Bloechle et al. (2015), we found that trajectories exhibited a leftward bias that was reduced for unit-decade incompatible comparisons. However, when positions of response labels were reversed, the biases reversed. That is, we found a rightward bias for compatible pairs that was reduced for incompatible pairs. This result calls into question a purely embodied representation of place value structure and instead supports a competition model of two-digit number representation.

Keywords: Two-digit comparison, place value, response competition, computer mouse tracking
\end{abstract}

Making mental comparisons of two-digit numbers can be a daily task for many people. For example, when driving an automobile, one must hold a fixed standard in memory (i.e., remembering that the speed limit is 65 miles per hour) while simultaneously perceiving a symbolic representation of the current speed from the speedometer and quickly deciding 
whether that speed is above or below the speed limit. Though this task is fairly automatic for practiced drivers, the cognitive mechanisms underlying such two-digit comparison are not well-understood. In the present paper, we examine the dynamics of how people compare two-digit numbers to a fixed standard.

The last 20 years have seen an incredible growth in our understanding of how people represent and process two-digit numbers. Accordingly, the literature has grown in complexity. There are two aspects of two-digit number cognition that we wish to address in the present paper. The first is the aspect of representation; that is, characterizing the link between perception and cognition that is formed when people perform tasks with two-digit numbers. The second is the aspect of processing; that is, characterizing the link between cognition and action that results during the decision-making process in a two-digit number task. While these two aspects are quite linked, we will first describe the relevant literature for each separately.

\section{Models of two-digit number representation}

Three competing models of two-digit number representation have emerged. One of the earliest attempts to understand the representations formed in two-digit number comparison was Hinrichs, Yurko, and Hu (ए98】), who asked participants to compare a two-digit number to a fixed standard (e.g., 55). They found that reaction times decreased as numerical distance from the standard increased. Going back to Moyer and Landauer (匹967), this numerical distance effect has been considered a signature of an analog representation of number. Put another way, this distance effect implies that "the internal representation of number may be an 'analog' of some physical property and that the number comparison process is similar to an internal psychophysical comparison process" (Hinrichs et al., 1.981, p. 890). In addition, Hinrichs et al. (1981) found that the unit digit (e.g., the " 8 " in the number 28) continued to affect processing times, even though paying attention to only the decade digit (e.g., the "2" in 28) is sufficient to make the comparison decision. This inability of participants to ignore the unit digit led Hinrichs et al. (एप, to conclude that two-digit

Please address correspondence to Thomas J. Faulkenberry, Department of Psychological Sciences, Tarleton State University, Box T-0820, Stephenville, TX 76402. Email: faulkenberry@tarleton.edu. All raw data and analysis code are available at https://git.io/v6AVI

The author would like to thank Brie Heidingsfelder, Jonathan Herring, Heather Hill, and Kate Shaw for their assistance with data collection.

Draft version: February 7, 2017 
numbers are represented as holistic, integrated quantities.

In a similar experiment, Dehaene, Dupoux, and Mehler (19.90) also found that reaction times decreased as numerical distance from the standard increased. Further, Dehaene et al. (1990) found that presenting the unit digit before the decade digit did not increase or decrease the influence of the unit digit on reaction times. Dehaene et al. ([1990) concluded that symbolic two-digit numbers must first be encoded into an internal, analog magnitude code on a mental number line (e.g., Restle, 1970; Moyer \& Landauer, 1967). In other words, early evidence pointed to the claim that skilled adults collapse the base-10 structure of a two-digit number into a single, holistic magnitude code.

However, Nuerk, Weger, and Willmes (2001) challenged this claim on the basis of the unit-decade compatibility effect. Such an effect occurs when it takes longer to choose the larger of the pair 36_52 than to choose the larger of 52_68. One possible reason for this is because in the pair 36_52, separate comparisons of the decades $(3<5)$ and the units $(6>2)$ lead to opposite decisions (thus 36_52 is called a unit-decade incompatible pair). On the other hand, for the pair 52_68, separate comparisons lead to the same decision $(5<6$ and $2<8)$, so $52 \_68$ is called a unit-decade compatible pair. As the overall distance between numbers in each pair is 16 , the presence of such a compatibility effect presents problems for a purely holistic account, which would predict no difference in reaction times between the two pairs. In addition to this compatibility effect, several researchers (e.g., Nuerk et al., 2001; Nuerk, Kaufmann, Zoppoth, \& Willmes, 2004; Wood, Nuerk, Freitas, Freitas, \& Willmes, 20106) found that overall distance predicted reaction times, leading Nuerk and Willmes (2005) to propose a hybrid model, where two-digit numbers activate both holistic and decomposed representations in parallel.

In addition to the holistic and hybrid models of two-digit number representation, there is a third class of models which posits that two-digit numbers are decomposed so that the magnitude of each digit is represented separately, with no integrated holistic representation (e.g., McCloskey, 1992; Verguts \& De Moor, 20115). Verguts and De Moor (2010.5) found evidence for such a decomposed model by manipulating overall distance (small versus large) and decade distance (same decade, different decade). They found that for same decade pairs, there was a numerical distance effect; that is, large distance pairs took less time to compare than small distance pairs. However, no such distance effect emerged for different decade pairs, presumably due to the fact that the larger of a different decade pair can be selected based on a comparison of the decade digit alone. Verguts and De Moor (2005) interpreted their results in terms of a model of single-digit number comparison (Verguts, Fias, \& Stevens, 200.5), arguing that decomposed representations allow recycling of efficient single-digit number processing to solve the inherently harder problem of two-digit number comparison. 


\section{Models of numerical decision processes}

Whereas the "holistic versus decomposed" debate has dominated the literature on twodigit number representation, much of the literature on single-digit numbers has focused the nature of the decision processes involved in number tasks. For example, Song and Nakayama (2008) had participants choose whether a single digit number was less than or greater than 5 by reaching and touching a square on a computer screen that was positioned either leftward or rightward from the centrally presented target number. Numbers smaller than 5 were identified by touching the left square, whereas numbers larger than 5 were identified by touching the right square. Critically, Song and Nakayama measured the streaming threedimensional coordinates of the participants' hand positions throughout the reaching motion on each trial, effectively providing a continuous index of participants' decision dynamics. They found that trajectories became more curved toward the center of the screen as the target numbers became closer in magnitude to the comparison standard (e.g., trajectories for the target 4 were more curved than trajectories for the target 1). Song and Nakayama (2008) argued that the increase in curvature obtained in such a number comparison task reflects a direct mapping from an internal "mental number line" to external space. Their explanation was that with numbers that are close to the comparison standard, the hand is drawn toward the external projection of the stimulus number, which would necessarily be represented closer to the middle of the mental number line. This would result in the appearance of more curvature in the response trajectories. Numbers that are far from the standard would be represented closer to the ends of the mental number line, and hence, their external projections would draw the hand more directly toward the response option (resulting in less curvature).

Santens, Goossens, and Verguts (सणU) argued instead that the increase in trajectory curvature that Song and Nakayama (20108) found for close number pairs is due to increased competition between parallel and partially active response options, basing their explanation on a computational model of number representation (Verguts et al., 2010.5). This model posits that when participants compare a number to a fixed standard such as 5 , the "winning" response (less than 5 or greater than 5 ) is the one whose activation level rises to a threshold necessary to trigger manual response initiation. When the target number is far from 5 , the correct response unit accumulates activation quickly, resulting in a fairly direct path toward the correct response on the screen. However, when the target number is close to 5 , the representational overlap between the target and the standard feeds forward to the decision process, causing both response units to accumulate activation at similar rates. These response units are then both partially active for a longer duration, resulting in the appearance of a more curved reaching trajectory. Such curvature would then be an artifact of the competition involved in the decision process, not a direct mapping of the underlying 
representation to external space.

Santens et al. (201) also pointed out that while these two models (competition versus direct mapping) make identical predictions when the response mapping is congruent with a "left=small, right=large" spatial mapping, the two models would yield divergent predictions for trajectories in the incongruent response mapping (LARGER - SMALLER). Figure $\square$ displays these predictions for large (greater than 5) targets. The competition view, they argued, would predict that trajectory curvature would decrease as a function of numerical distance, since larger distance pairs would have less representational overlap, and hence less response competition, leading to less curvature toward the incorrect response option. This can be seen in Figure $\mathbb{D}$ by noting that for the competition view, (the bottom row), the dashed trajectories become less curved toward the middle as distance to the comparison standard increases from small to large. In contrast, the direct mapping view (top row) would predict that trajectory curvature would increase as a function of numerical distance, because with an incongruent response mapping, the external projection of a number that is far from the comparison standard would be at the opposite end of where the response is to be made. For example, consider Figure m: 9 would be projected to the far right of physical space, but due to the incongruent response mapping, the decision LARGER would be made on the left side of the screen. Hence, the initial hand movement would be toward the right, but the hand would have to move all the way to the left of the screen to make the response. This overall movement would have a large amount of curvature compared to a number whose external projection would be closer to the middle of the externally projected space (e.g., 6). In their paper, Santens et al. (2011) tested between these alternatives for single digit numbers and found that in the incongruent response mapping condition, curvatures decreased, supporting the competition view (see also Faulkenberry, 2016).

\section{Embodied numerical cognition}

In addition to the previously described debates on the nature of numerical cognition from the standpoints of representation and processing, there has been a parallel debate concerning how number concepts are stored in long-term memory. Recently, several researchers have reported evidence of associations between number concepts and the sensorimotor experiences obtained during numerical knowledge acquisition. For example, the spatial orientation of one's mental number line has been shown to depend upon factors such as reading and writing direction (Shaki, Fischer, \& Petrusic, 2009) and finger-counting habits (Fischer, $20108)$. In addition, bodily states can interplay with action initiation in number tasks. For example, Lindemann, Abolafia, Girardi, and Bekkering (2007) demonstrated that participants' hand grip openings were initiated faster and resulted in wider grip openings when responding to large numbers, compared to smaller grip openings for small numbers. Addi- 


\section{Small distance}

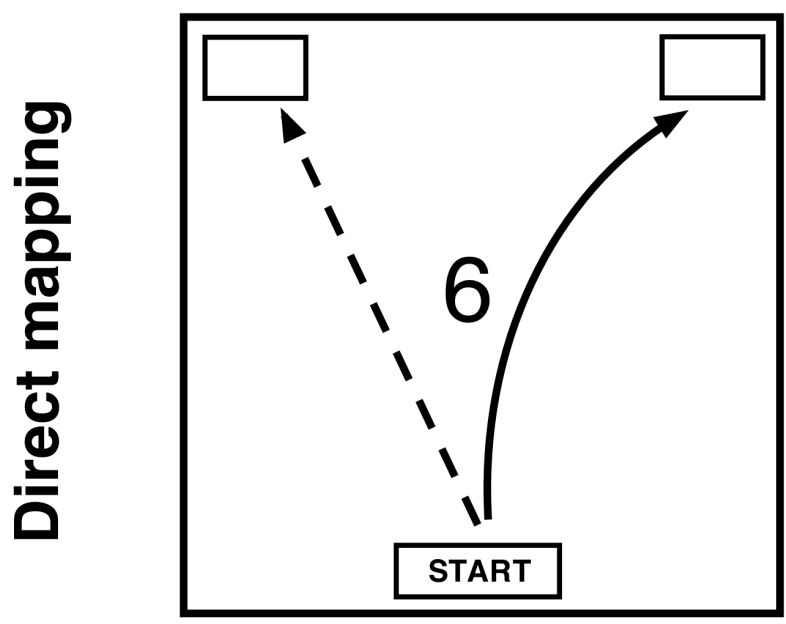

Large distance

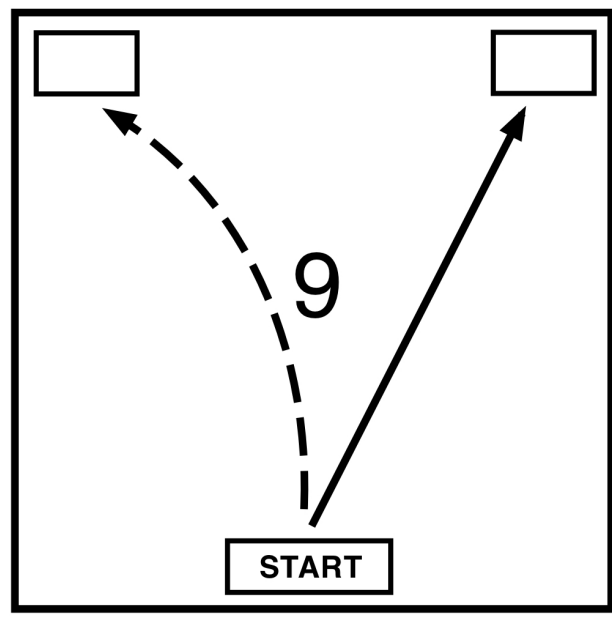

congruent mapping

$\rightarrow-\rightarrow$ incongruent mapping
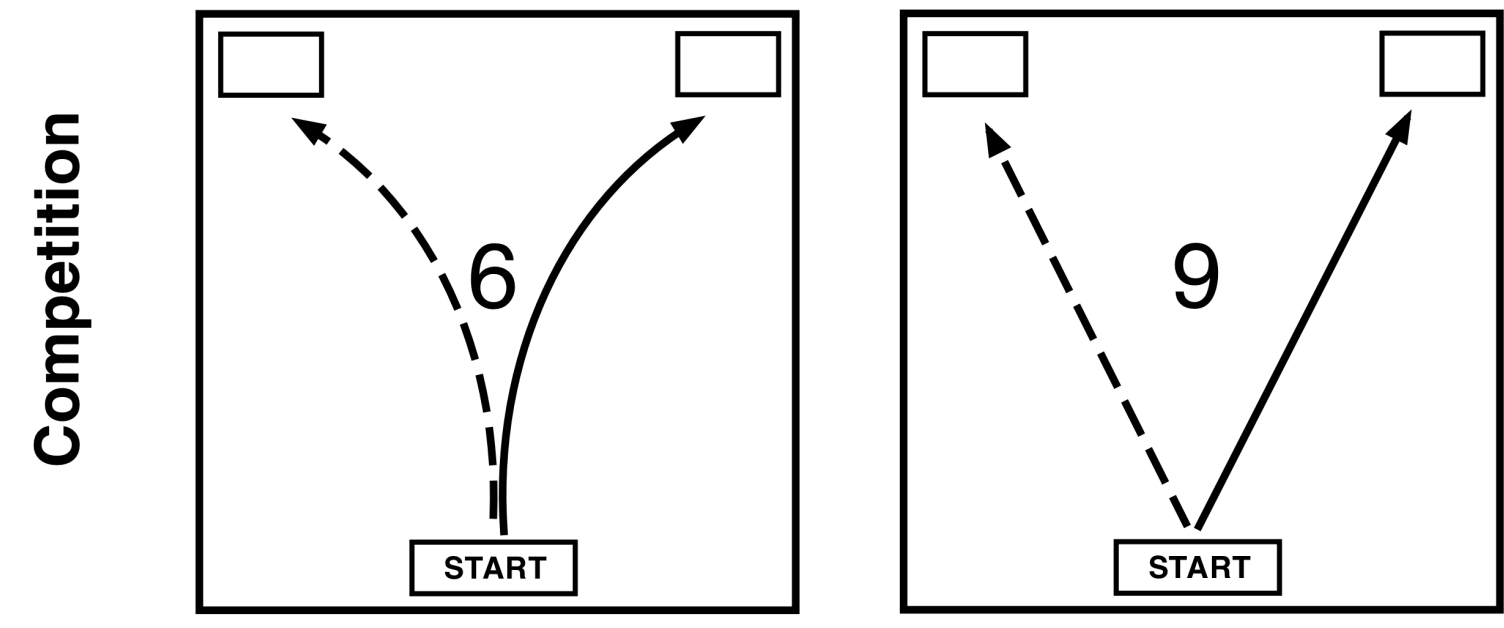

Figure 1. Idealized predicted trajectories toward large single-digit targets (greater than 5) from the direct mapping account (Song \& Nakayama, 2008) and the competition account (Verguts, Fias, \& Stevens, 2005), displayed as a function of numerical distance (small versus large) and response mapping (congruent versus incongruent). Figure adapted from Santens, Goossens, and Verguts (2010) and Faulkenberry (2016). 
tionally, grip closure was initiated faster for small numbers. Such interactions among bodily states, number representations, and number decisions can be accounted for by embodied theories of cognition (Barsalou, 2018), which broadly state that the peripheral sensory and motor systems are not only responsible for monitoring and executing bodily movements, but are also integral in the formation of knowledge representations.

Along this line, Bloechle et al. (2015) found evidence for a long-term association between space and number in the context of place-value for two-digit numbers. In their study, Bloechle et al. (2015) had participants point to the larger of a pair of two-digit numbers on a touch screen. They found that the landing position of the finger on the target number was significantly left of the midline between the decade and unit digit. Further, this "decade bias" was reduced on unit-decade incompatible trials, presumably because on such trials the interfering magnitude information from the unit digit resulted in increased unit processing. Bloechle et al. (2015) interpreted this response pattern as reflective of a longterm memory association of decades with leftward space and units with rightward space, possibly due to the past experience of reading and writing multi-digit Arabic numerals. They argued that such behavior reflects "an embodied representation of the place-value structure of two-digit numbers" (Bloechle et al., 2015, p. 480), presumably because reading and writing two-digit numbers results in repeated, systematic digit-space associations of "decade = left" and "unit = right".

To our knowledge, the study of Bloechle et al. (2015) is the only such study to find such a digit bias in manual pointing positions. However, we are aware of one other study that investigated the dynamics of manual responses in two-digit number comparison. Dotan and Dehaene (20113) tracked participants' finger trajectories as they pointed to the approximate location of a single two-digit number on a number line. Dotan and Dehaene (2013) concluded that when performing the number-to-position task, adults form a fast representation of the unit digit magnitude, followed by both holistic and decomposed representations of the twodigit number. Their results indicate that both holistic and decomposed representations play a role in two-digit number representation (supporting the hybrid model, e.g., Nuerk et al., 2001). Further, their data indicate that unit processing may override decade processing (at least through the first $550 \mathrm{~ms}$ of the response execution).

Given the inability of previous studies to discriminate among competing models of two digit number representation (holistic, decomposed, or hybrid), the studies of Bloechle et al. (2015) and Dotan and Dehaene (2013) are an important next step in such a research program, particularly because they explore the dynamics of the hand movements that people make when making decisions about two-digit numbers. Tracking these hand movements is becoming an increasingly popular method for testing various models in numerical cognition (Fischer \& Hartmann, 2014; Faulkenberry \& Rey, 2014). Because these hand movements 
reflect the projection of internal cognitive processes onto an observable behavioral output (Spivey, Grosjean, \& Knoblich, 20105; Spivey, 2007), such hand tracking provides a window into the internal representations that evolve during a numerical decision. Several recent studies have exploited this technique in various forms, such as directly tracking hand/finger movements (Song \& Nakayama, [018); Santens et al., 2010) and tracking the movement of a computer mouse (Marghetis, Núñez, \& Bergen, 2014; Faulkenberry, 2014; Ganor-Stern \& Goldman, 2014; Faulkenberry, Montgomery, \& Tennes, 2015; Haslbeck, Wood, \& Witte, 2015; Faulkenberry, Cruise, Lavro, \& Shaki, 2016; Faulkenberry, 2016).

\section{The present study}

The goal of the present study was to investigate the dynamics of two-digit number representations and the resulting decision processes. We asked participants to use a computer mouse to choose whether two-digit numbers were smaller or larger than 55 . Further, we had participants perform these comparison judgments in two response mapping conditions: one with the labels SMALLER - LARGER displayed (a spatially congruent response mapping), and the other with LARGER - SMALLER displayed (a spatially incongruent response mapping). To measure the dynamics of participants' decision processes, we recorded the streaming positions of the mouse cursor on the computer screen. Our primary goal was to explore the dynamic processing signatures of holistic representations (i.e., a single integrated representation of number magnitude) and decomposed representations (i.e., separate representations of digit magnitude) and test competing explanations for these signatures. Signatures for the dynamic influence of numerical magnitude (such as increased curvature with decreasing numerical distance) have already been found in multiple studies with single digit numbers (Song \& Nakayama, 2008; Santens et al., 2011; Faulkenberry, 2016). We generalize these results to two-digit numbers by predicting that computer mouse trajectories will become more curved toward the middle of the computer display as the distance between the target number and the comparison standard 55 decreases. Further, by manipulating the spatial congruity of the response labels as in Santens et al. (खणI) and Faulkenberry (2016), we can test whether this curvature is due to a direct mapping of target numbers onto an externally projected number line (e.g., Song \& Nakayama, 2010$)$, or rather due to increased indecision from response competition due to increasing representational overlap between the target and the comparison standard.

For decomposed representations, the nature of such processing signatures is less clear. As already mentioned, we are aware of only two other studies that have investigated manual responses in two-digit number comparison, and only one of these (Dotan \& Dehaene, [2013) tracked the continuous dynamics of the manual response. Both Bloechle et al. (2015) and Dotan and Dehaene (2013) have found a response bias reflecting increased activation of a 
particular digit. Also, Bloechle et al. (2015) found that on unit-decade incompatible trials, the bias toward the decade digit was reduced, implying a greater activation of the unit digit. As such, we predict to obtain a similar result with mouse trajectories. However, it is not clear at present what the source of this bias may be. Though Bloechle et al. (2015) explained that such bias may be the result of long term memory associations between digits and space, (e.g., a direct mapping account), we predict that such bias may be the result of competition between parallel and partially active response options (Santens et al., 2011; Faulkenberry, 2016). That is, if the manual digit bias observed in Bloechle et al. (2015) and Dotan and Dehaene (2013) is due to a direct mapping stemming from a long-term association of place value and the spatial arrangement of decade=left, unit=right, then trajectories should exhibit less leftward bias on unit-decade incompatible trials; critically, this reduction of bias should not depend on the type of response mapping used. On the other hand, if the manual digit bias is due to response competition (Santens et al., 2011; Faulkenberry, 2016), then the bias patterns (e.g., reduction of leftward bias on unit-decade incompatible trials) would be due to increasing activation of the competing response option, and hence the bias patterns should reverse when using the spatially incongruent response mapping (LARGER - SMALLER). Such reversal would indicate that the unit digit bias observed in Bloechle et al. (2015) and Dotan and Dehaene (2013) may be due to task demands rather than an inherently embodied representation of place value.

\section{Method}

\section{Participants}

Thirty-seven undergraduate students (36 female, mean age $=24.1$ years, age range 18 to 60) participated in this experiment in exchange for partial course credit in their psychology courses. Four participants reported being left hand-dominant, but all reported that they used their right hand for the computer mouse. $\mathrm{m}$ The experiment was reviewed and approved by the institutional review board at Tarleton State University.

\section{Apparatus}

The experiment was implemented using the MouseTracker software package (Freeman \& Ambady, [UणU). The experimental trials were presented on a 20 inch iMac desktop computer with a screen resolution of 1,280 x 960 pixels. We ran the MouseTracker program on the iMac using a virtual Windows XP environment via Parallels. Following the recommendations of Fischer and Hartmann (2014), we disabled the "dynamic acceleration" option and lowered the speed of the mouse movements on the screen to the second-lowest possible

\footnotetext{
${ }^{1}$ We performed a separate analysis where we excluded the 4 left-handed participants. We found exactly the same pattern of results for both analyses.
} 
speed in the mouse settings dialog. This is done to prevent quick and erratic mouse movements, resulting in a smooth and more reliable record of participants' hand movements. The resulting displacement ratio of the mouse to screen movement was $1 \mathrm{~cm}$ to 100 pixels.

The experimental interface was designed in a manner similar to other recent mousetracking experiments (e.g., Faulkenberry, 2014; Faulkenberry et al., 2015; Faulkenberry, 2016; Faulkenberry et al., एण16). The visible part of the computer display was a rectangle with height $27 \mathrm{~cm}$ and width $36 \mathrm{~cm}$, colored in medium gray, RGB $=(160,160,160)$. Two rectangular response boxes (height $=27 \mathrm{~mm}$, width $=54 \mathrm{~mm}$ ) were placed at the upper left and right corners. Both response boxes were solid black with response labels written in white 24 point Arial font. A single black rectangular button (height $=18 \mathrm{~mm}$, width $=36 \mathrm{~mm}$ ) was placed in the bottom center of the screen with the text "START" centered and written in white 18 point Verdana font. The start button was equidistant from both response labels $(32.4 \mathrm{~cm}$ from bottom center to upper corner). Given the mouse-to-pixel displacement ratio reported above, participants needed to move the computer mouse approximately $11.4 \mathrm{~cm}$ on the table in order to move the cursor along a straight path from start to response. Stimulus numbers (see below) were presented in the center of the screen and written in black 64 point Arial font.

\section{Stimuli and procedure}

Our stimulus set consisted of 64 two-digit numbers adapted from Reynvoet, Notebaert, and Van den Bussche (2010). We asked participants to perform a two-digit number comparison using 55 as the comparison standard. ${ }^{\square}$ We excluded all pure decade numbers (e.g., 60) and tie numbers (i.e., numbers where unit and decade digits were equal, such as 44). We manipulated size (smaller than 55, larger than 55), holistic distance (close to 55, far from 55), and unit-decade compatibility (compatible, incompatible). We defined "close" and "far" holistic distance as follows: for a given compatibility condition, we divided the set of 16 numbers that were less than 55 into two sets of 8 numbers. The 8 smallest were labeled "far" and the 8 largest were labeled "close". Similarly, we divided the 16 numbers greater than 55 into two sets of 8 , labeling the 8 largest as "far" and the 8 smallest as "close". See Table $\mathbb{\square}$ for the complete list of stimuli.

Participants were told that on every trial a number would appear in the center of the screen, and they would be asked to choose, as quickly as possible, whether the number was less than 55 or greater than 55. Each trial started with a blank screen presented for $500 \mathrm{~ms}$, followed by a screen that displayed the response labels SMALLER and LARGER at the top left and right of the screen, respectively, as well as the START button. The

\footnotetext{
${ }^{2}$ Using a fixed standard mirrored the design of previous manual response tracking studies and allowed us to easily manipulate spatial response congruity by simply switching the position of response labels (e.g., Santens et al., 201]; Faulkenberry, 2016).
} 
Table 1

Two-digit stimuli used in the comparison task

\begin{tabular}{|c|c|c|}
\hline & \multicolumn{2}{|c|}{ Holistic distance } \\
\hline & Close & Far \\
\hline \multicolumn{3}{|c|}{ Unit-decade compatible targets } \\
\hline Less than 55 & 3132343541424345 & 1213141521232425 \\
\hline Greater than 55 & 5657585965676869 & 7576787985868789 \\
\hline \multicolumn{3}{|c|}{ Unit-decade incompatible targets } \\
\hline Less than 55 & 3637383946474849 & 1617181926272829 \\
\hline Greater than 55 & 6162636471727374 & 8182838491929394 \\
\hline
\end{tabular}

Note. Mean distance from 55: close $/$ compatible $=12.25$, close $/$ incompatible $=12.5$, far $/$ compatible $=31.75$, far $/$ incompatible $=32.5$

order of these response labels was switched once midway through the experiment; half of the participants started with the SMALLER-LARGER ordering, while the other half began with the LARGER-SMALLER ordering. Once the START button was pressed, one of the stimulus numerals appeared in the center of the screen. Beginning from the center of the start button, participants moved the computer mouse and then clicked on the correct of these two options; while doing so, the software recorded the streaming $(x, y)$ coordinates of the computer mouse approximately 70 times per second. The mouse pointer was automatically relocated to the center of the Start button at the end of each trial.

We manipulated the spatial congruity of the response labels SMALLER and LARGER: in the congruent condition, SMALLER appeared in the upper left corner and LARGER appeared in the upper right corner. In the incongruent condition, these labels were reversed. In half of the trials, the correct answer was on the left side, whereas on the other half of the trials, the correct answer was on the right side.

For incorrect responses, the program displayed an " $\mathrm{X}$ " for $1000 \mathrm{~ms}$. To ensure that trajectories reflected online processing, participants were encouraged to begin their movements as early as possible and were warned if initiated movement later than $400 \mathrm{~ms}$ following stimulus presentation. This instruction is customarily included in mousetracking studies so that trajectories reflect the dynamics of a decision process rather than simply reflecting the kinematics of a response choice after the choice has already been made (Freeman \& Ambady, 20109; Spivey et al., 201015).

For each of the two counterbalanced spatial congruity conditions, participants completed two blocks of 64 trials (2 repetitions of each stimulus number, randomly presented), 
with a short break in between each block. In all, each participant completed 256 experimental trials in a single 30 minute session.

\section{Results}

\section{Data preparation and analysis plan}

Participants completed a total of 9,472 experimental trials. Of these, we removed 40 trials that contained an error response $(0.42 \%)$. Over the remaining correct trials, participants exhibited a mean response time of $1198 \mathrm{~ms}(\mathrm{SD}=356 \mathrm{~ms})$. Each manual response consisted of an initiation time (time to initiate mouse movement after pressing the start button; $M=118 \mathrm{~ms}, S D=93 \mathrm{~ms}$ ) and movement time (time span from movement initiation to response; $M=1080 \mathrm{~ms}, S D=362 \mathrm{~ms}$ ). We removed an additional 168 trials for which response time exceeded 3 standard deviations from the mean response time, as well as any trials for which response time was less than $200 \mathrm{~ms}$ (5 trials). All subsequent analyses were performed on the remaining 9,259 trials.

All hypotheses were tested with $\mathrm{R}$ ( $\mathrm{R}$ Development Core Team, 201]) using the general linear model with $\alpha=0.01$. Thus, all ANOVA models were compared against a null model using a critical $F$-value of $F_{\text {crit }}(1,36)=7.39$. We chose to use a value of $\alpha$ smaller than the conventional $\alpha=0.05$ for two reasons. First, a lower $\alpha$ lessens the risk of a false-positive, or Type I error. Second, $p$-values between 0.01 and 0.05 often carry small evidential value (Wagenmakers, 2007 ), particularly when $p$-values are between 0.04 and 0.05. In such cases, the likelihood of the null hypothesis can actually far outweigh the likelihood of the alternative, a finding known as Lindley's paradox (Lindley, 1957). On the contrary, by requiring any "significant" effect to have an $F$-value at least 7.39 , we are requiring a larger threshold of evidence for any putative effect. Indeed, one can use approximate Bayes factors (Masson, 2011; Faulkenberry \& Tummolini, 2016) to calculate that if $F>7.39$, then the model including the effect is 5.15 times more likely than the null model, a ratio generally considered as moderate evidence (Jeffreys, ए961).

\section{Holistic processing signatures}

Time analyses. Mouse movement times (MT) were submitted to a 2 (Distance: close, far) x 2 (Response side: left, right) x 2 (Response mapping: congruent, incongruent) repeated measures analysis of variance. There was a significant main effect of distance,

$F(1,36)=123.1, p<0.001, \eta_{p}^{2}=0.77$. As can be seen in Figure $\boldsymbol{\nabla}$, movement times were longer for two-digit numbers that were close to 55 ( $M=1085 \mathrm{~ms})$ compared to trials with numbers far from $55(M=1024 \mathrm{~ms})$. No other terms in the ANOVA model were statistically significant (all $F$-values less than 0.89 ). 
Similarly, initiation times were submitted to a 2 (Distance: close, far) x 2 (Response side: left, right) x 2 (Response mapping: congruent, incongruent) repeated measures analysis of variance. No terms in the ANOVA model were statistically significant (all $F$-values less than 4.80).

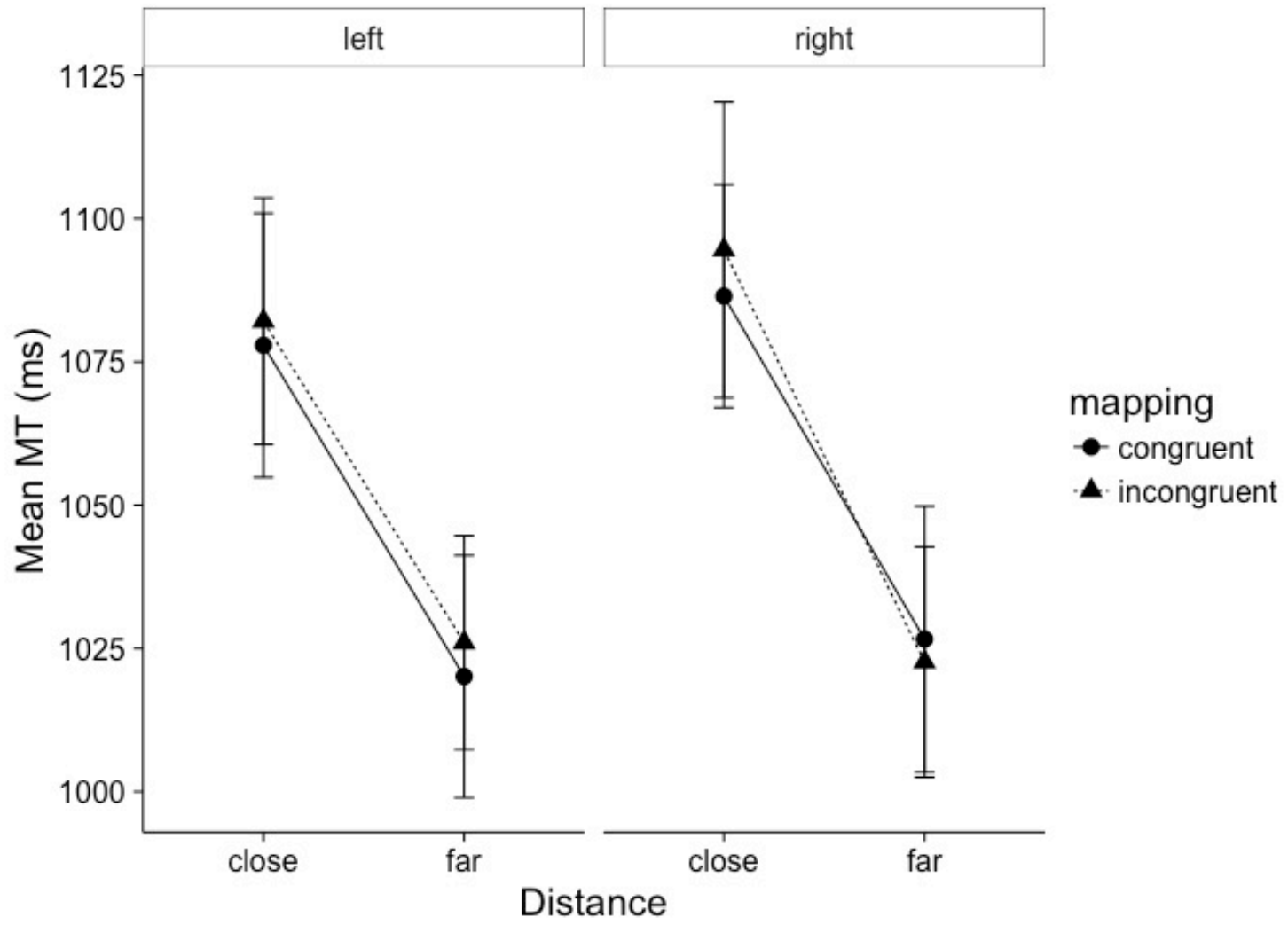

Figure 2. Mean movement times as a function of distance (close, far), response side (left, right), and response mapping (congruent, incongruent). Error bars represent within-subject $95 \%$ confidence intervals as recommended by Morey (2008).

Trajectory analyses. Mouse movement trajectories were measured by recording the streaming $x, y$ - coordinates of the computer mouse during each trial. In order to compare trajectory characteristics across trials of differing response times, all raw mouse trajectories were pre-processed in MouseTracker (Freeman \& Ambady, 2010) so that all trajectories were rescaled onto a standard coordinate space of $[-1,1] \times[0,1.5]$ and normalized via linear interpolation to consist of exactly 101 timesteps.

In Figure [1, we present average mouse trajectories as a function of distance (close, far), response mapping (congruent, incongruent), and decision (larger than 55, smaller than 55). As can be seen in the figure, trajectories are more curved toward the incorrect response alternative for numbers that are close to the comparison standard of 55, compared 
to trajectories for numbers that are far from 55. This increase in trajectory curvature is in line with patterns reported in several previous studies (Song \& Nakayama, 20108; Santens et al., 2011).

As mentioned earlier, there are two possible explanations for this increased trajectory curvature, a direct mapping view (Song \& Nakayama, 2008) and a competition view (Santens et al., 2010; Faulkenberry, 2016). Recall that the discriminating signature comes from the pattern of response curvatures with distance in the incongruent response mapping; increasing curvatures with distance support the direct mapping view, whereas decreasing curvatures with distance support the competition view. To test this, we computed area under the curve (AUC) as an index of curvature for each trajectory. measures above, AUC values were submitted to a 2 (Distance: close, far) x 2 (Response side: left, right) x 2 (Response mapping: congruent, incongruent) repeated measures analysis of variance. As can be seen in Figure $\mathbb{\theta}$, there was a significant main effect of distance, $F(1,36)=81.4, p<0.001, \eta_{p}^{2}=0.69$. AUC values for numbers that were close to the comparison standard were larger $(M=0.68)$ than for numbers which were far from the comparison standard $(M=0.45)$. No other terms in the ANOVA model were statistically significant (all $F$-values less than 3.8). Critically, the lack of an interaction between distance and response mapping $(F(1,36)=0.99, p=0.33)$ implies that the pattern of decreasing curvatures as a function of distance holds when restricted to incongruent trials. That is, the pattern of decreasing curvature with increasing distance does not differ as a function of response mapping, and thus holds for both congruent and incongruent trials. To confirm this, we performed a separate analysis of AUC values using a 2 (Distance: close, far) x 2 (Response side: left, right) repeated measures analysis of variance restricted to incongruent trials only. The main effect of distance remained significant, $F(1,36)=52.8, p<0.001$, $\eta_{p}^{2}=0.59$. There was no main effect of response side or interaction between distance and side (both $F$-values less than 0.44 ). This result supports the competition model over the direct mapping model. Whereas the distance effect reflects a basic signature of holistic representation (at least at the input level), the hand movements reflect a competition between competing response nodes instead of a direct mapping from an internal mental number line to external space.

\footnotetext{
${ }^{3}$ The MouseTracker software computes two measures of deflection - the area under the curve (AUC) as well as maximum deviation (MD). We performed analyses with MD as well and found exactly the same pattern of results. Since AUC is a priori a better measure of overall attraction toward the unselected result (Freeman \& Ambady, 20IU), we think it is a more appropriate measure of response competition, and is thus our choice for indexing trajectory curvature in this paper.
} 


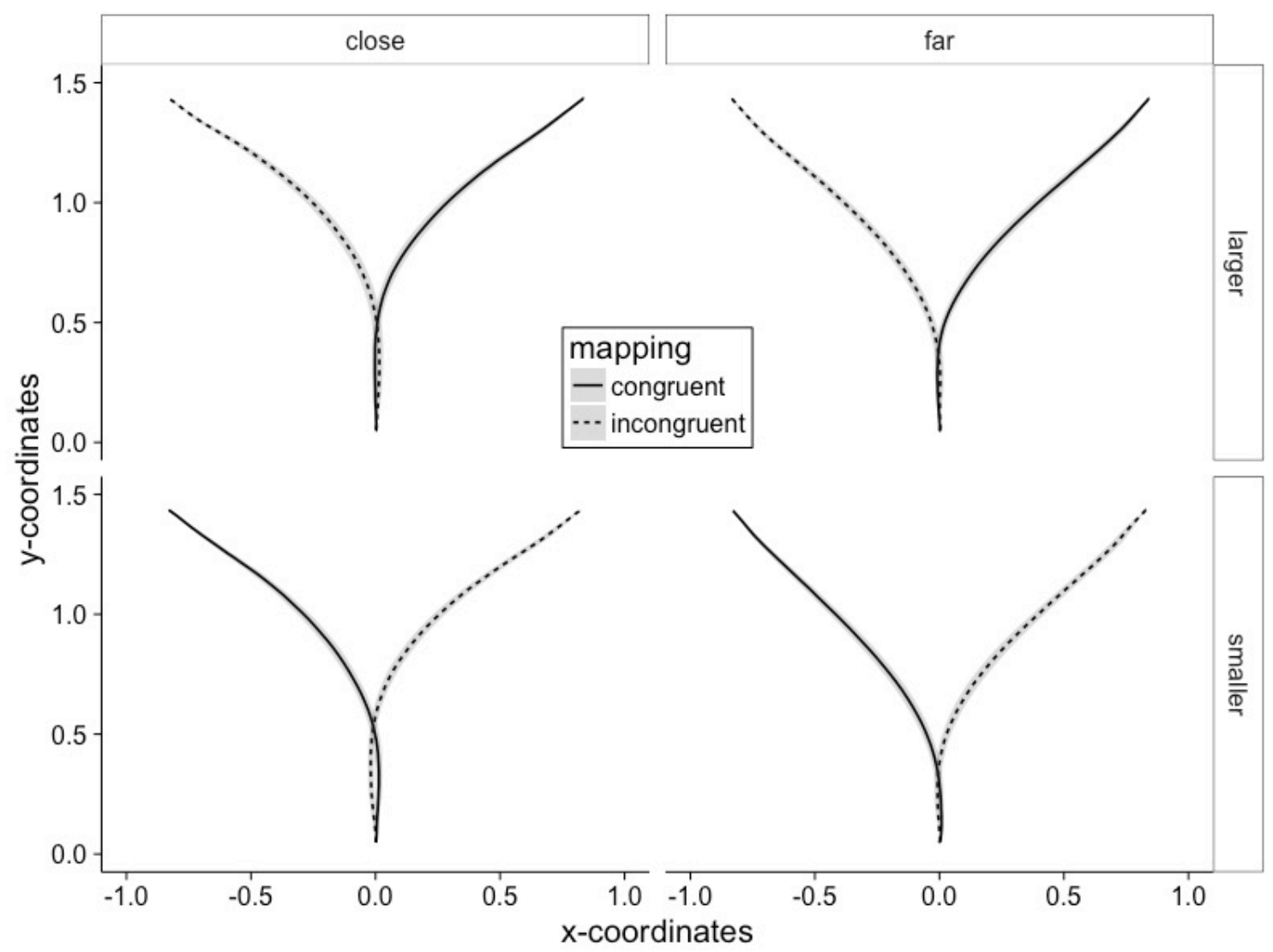

Figure 3. Average computer mouse trajectories as a function of distance (close, far), response mapping (congruent, incongruent), and decision (larger than 55, smaller than 55). Shading represents one standard error, computed from the mean $x$-coordinates of trajectories over the sample of 37 participants.

\section{Decomposed processing signatures}

Time analyses. Mouse movement times (MT) were submitted to a 2 (Unit-decade compatibility: compatible, incompatible) x 2 (Response side: left, right) x 2 (Response mapping: congruent, incongruent) repeated measures analysis of variance. There was a significant main effect of unit-decade compatibility, $F(1,36)=17.9, p<0.001, \eta_{p}^{2}=0.33$. As shown in Figure 1 , movement times for incompatible trials were slightly faster $(M=1044$ $\mathrm{ms}$ ) than compatible trials $(M=1065 \mathrm{~ms})$. There was a significant three-way interaction between compatibility, response mapping, and response side, $F(1,36)=24.3, p<0.001$, $\eta_{p}^{2}=0.40$. There was an interaction between Unit-decade compatibility and response mapping for rightward trajectories, $F(1,36)=19.4, p<0.001, \eta_{p}^{2}=0.35$, but the interaction did not quite reach our a priori threshold of significance for leftward trajectories, $F(1,36)=7.3$, $p>0.01$. 


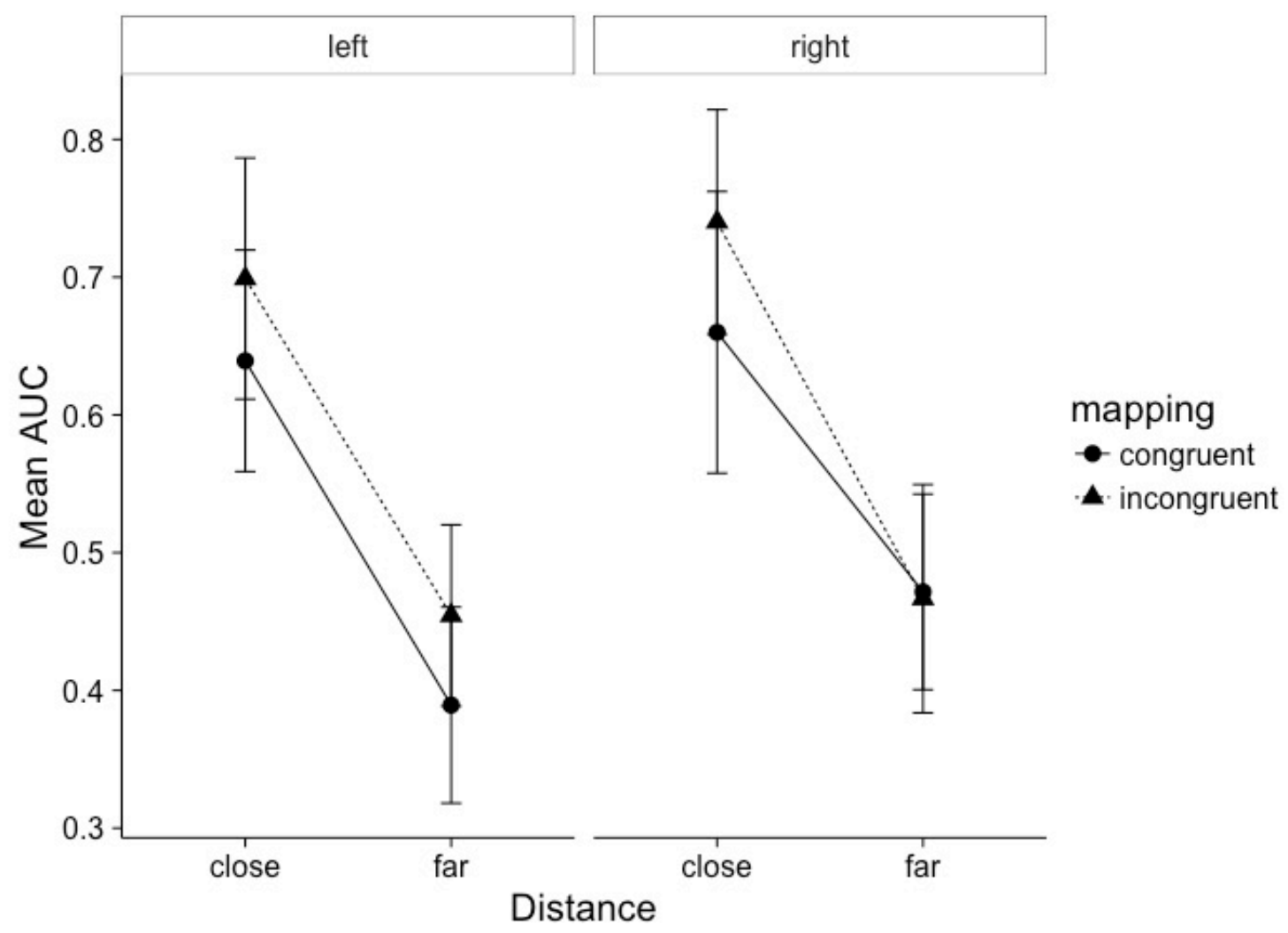

Figure 4. Mean AUC values as a function of distance (close, far), response side (left, right), and response mapping (congruent, incongruent). Error bars represent within-subject $95 \%$ confidence intervals as recommended by Morey (2008).

We also submitted initiation times to a 2 (Distance: close, far) x 2 (Response side: left, right) x 2 (Response mapping: congruent, incongruent) repeated measures analysis of variance. No terms in the ANOVA model were statistically significant (all $F$-values less than 1.0).

Trajectory analyses. In Figure 6, we present average mouse trajectories as a function of unit-decade compatibility (compatible, incompatible), response mapping (congruent, incongruent), and response side (left, right). To index trajectory curvatures, we submitted AUC values to a 2 (Unit-decade compatibility: compatible, incompatible) x 2 (Response side: left, right) x 2 (Response mapping: congruent, incongruent) repeated measures analysis of variance. No main effects or two-way interactions in the ANOVA model were significant (all $F$-values less than 3.9). However, as we can see in Figure $\mathbb{Z}$, there was a significant three-way interaction between compatibility, response mapping, and response side, $F(1,36)=52.7, p<0.001, \eta_{p}^{2}=0.59$. As shown in Figures [ 6 and $\square$, there was an interaction between compatibility and response side for congruent trials, $F(1,36)=26.5, p<0.001$, 


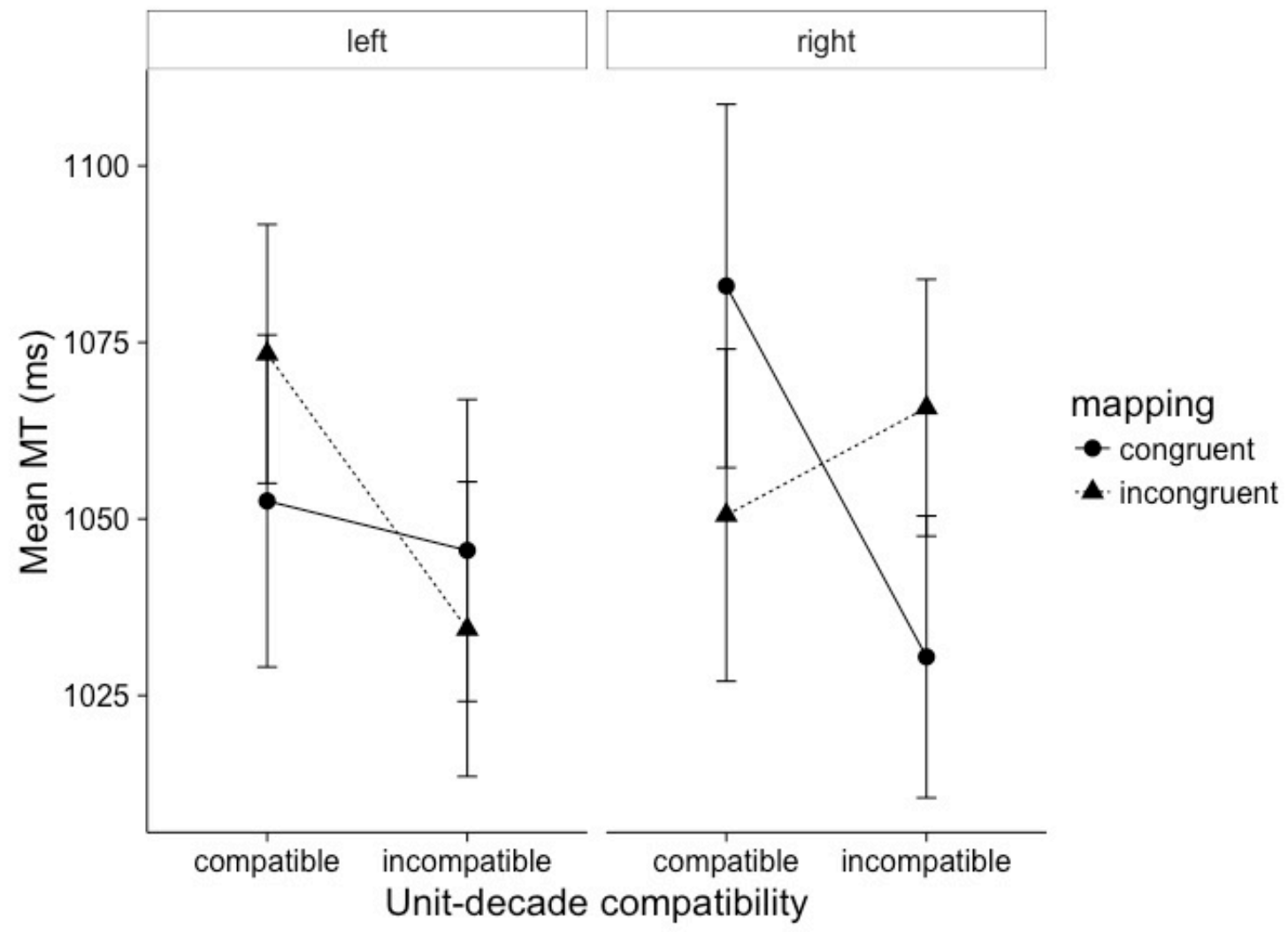

Figure 5. Mean movement times as a function of unit-decade compatibility (compatible, incompatible), response side (left, right), and response mapping (congruent, incongruent). Error bars represent within-subject $95 \%$ confidence intervals as recommended by Morey (20108).

$\eta_{p}^{2}=0.42$. For trials in the congruent response mapping (SMALLER - LARGER), trajectories exhibited reduced leftward bias on incompatible trials. This is indexed by a smaller AUC value for incompatible trials in rightward trajectories (compatible, $M=0.65$; incompatible, $M=0.49$ ) and a larger AUC value for incompatible trials in leftward trajectories (compatible, $M=0.50$; incompatible, $M=0.53$ ). Such a result reflects increased activation of the unit digit for incompatible trials throughout the response process, which is consistent with the findings of both Bloechle et al. (2015) and Dotan and Dehaene (2013).

One explanation for this unit bias on incompatible trials may be that the unit digit is the one whose decomposed comparison is opposite of the correct decision. Such inconsistency may result in increased attention being dedicated to this digit (Moeller, Fischer, Nuerk, \& Willmes, 200.9). As Bloechle et al. (2015) suggest, this increased attention may lead to a direct interaction between basic representation of place value and motor processing (e.g., an embodied, direct mapping view), leading to the hand being drawn rightward 
(toward the spatial position of the unit digit) on these trials. On the other hand, the bias may be due to increased competition between response nodes (Santens et al., 2011; Faulkenberry, (2016). If the unit bias we observed on incompatible trials is the result of an embodied representation of place value, then we should see the same rightward bias on incompatible trials even with an incongruent response mapping (LARGER - SMALLER).

On the other hand, if the unit bias is the result of competition, then the unit bias should be reversed with an incongruent response mapping. That is, trajectories should be drawn leftward instead of rightward. As can be seen in Figure $\square$, this is exactly what we found. For incongruent trials, there was a significant interaction between compatibility and response side, $F(1,36)=22.3, p<0.001, \eta_{p}^{2}=0.38$. Rightward trajectories exhibited a larger AUC value for incompatible trials than for compatible trials. But, leftward trajectories exhibited a smaller AUC value for incompatible trials than for compatible trials. Both of these patterns are evidence for a leftward shift in trajectories on incompatible trials. Such a shift cannot be explained by the embodied perspective of Bloechle et al. (2015), and rather reflects an interaction between motor processing and place value that is tied to specific task demands.

\section{Discussion}

The purpose of the present study was to examine the dynamics of adults' two-digit number representations. Using computer mousetracking, we tracked and explored the characteristics of participants' hand movements as they chose whether presented two-digit numbers were either larger or smaller than 55. Our participants showed evidence of both holistic and decomposed representations. First, we saw that trajectories became more curved toward the incorrect response as targets became numerically closer to the comparison standard of 55. This dynamic numerical distance effect extends the findings of several recent experiments with single digit number comparison (Song \& Nakayama, 2018; Santens et al., 2011; Faulkenberry, 2016) and implies that participants are forming holistic representations when making magnitude decisions about two-digit numbers. Further, we found evidence of decomposed processing by showing that trajectories for unit-decade incompatible exhibited a directional bias that was modulated by response mapping. In all, these results support a hybrid model of two-digit number processing (e.g., Nuerk \& Willmes, 2005).

In addition to providing evidence supporting the hybrid model of Nuerk and Willmes (2005), we were able to go further and test between competing explanations of some recent results with response dynamics in two-digit number comparison. With regard to holistic magnitude processing, some researchers have claimed that response dynamics in a comparison task reflect a direct mapping between response effectors (e.g., hands or fingers) and a mental number line (Song \& Nakayama, 2018). However, using a line of reasoning simi- 


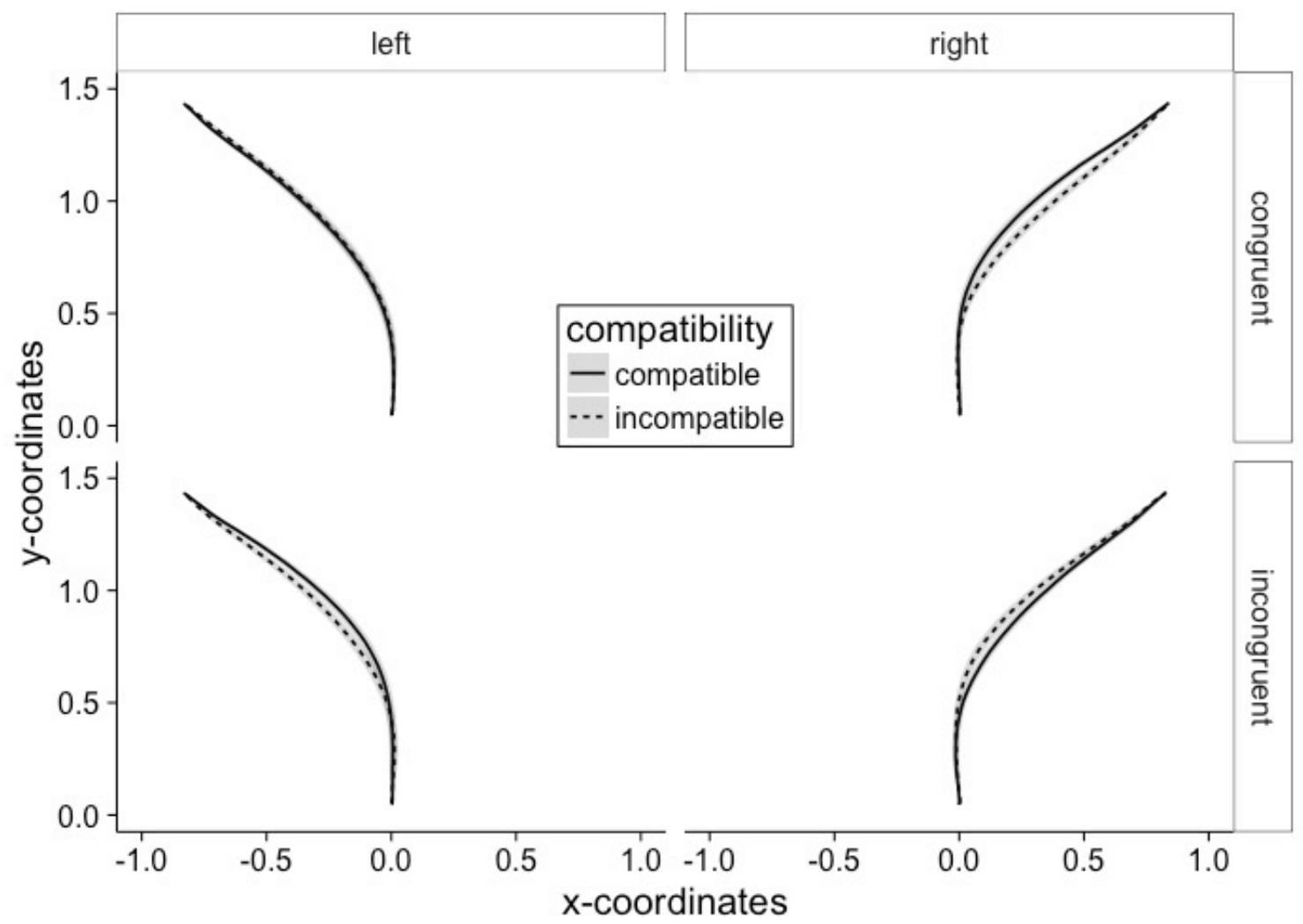

Figure 6. Average computer mouse trajectories as a function of unit-decade compatibility (compatible, incompatible), response mapping (congruent, incongruent), and response side (left, right). Shading represents one standard error, computed from the mean $x$-coordinates of trajectories over the sample of 37 participants.

lar to Santens et al. (2010) and Faulkenberry (2016), we showed that response curvatures increased with decreasing comparison distance regardless of the response mapping used. Such a result is not predicted by the direct mapping model and instead supports a competition model of number processing (e.g., Verguts et al., 2005). In this model, response times (and by extension, manual response curvatures) for spatially incongruent responses increase because incoming number stimuli activate a conceptual spatial code (e.g., small or large) which then feeds the incorrect response code, causing competition at the response level. The idea that middle-level representational structures are responsible for these compatibility effects is also reflected in the work of Proctor and Cho (2006), who also argued that such interference happens at a level between stimulus and response. Though these existing computational models are not specifically designed for two-digit number tasks, our results indicate that the model of Verguts et al. (2010.5) is at least conceptually plausible to explain magnitude processing in the two-digit number case. 


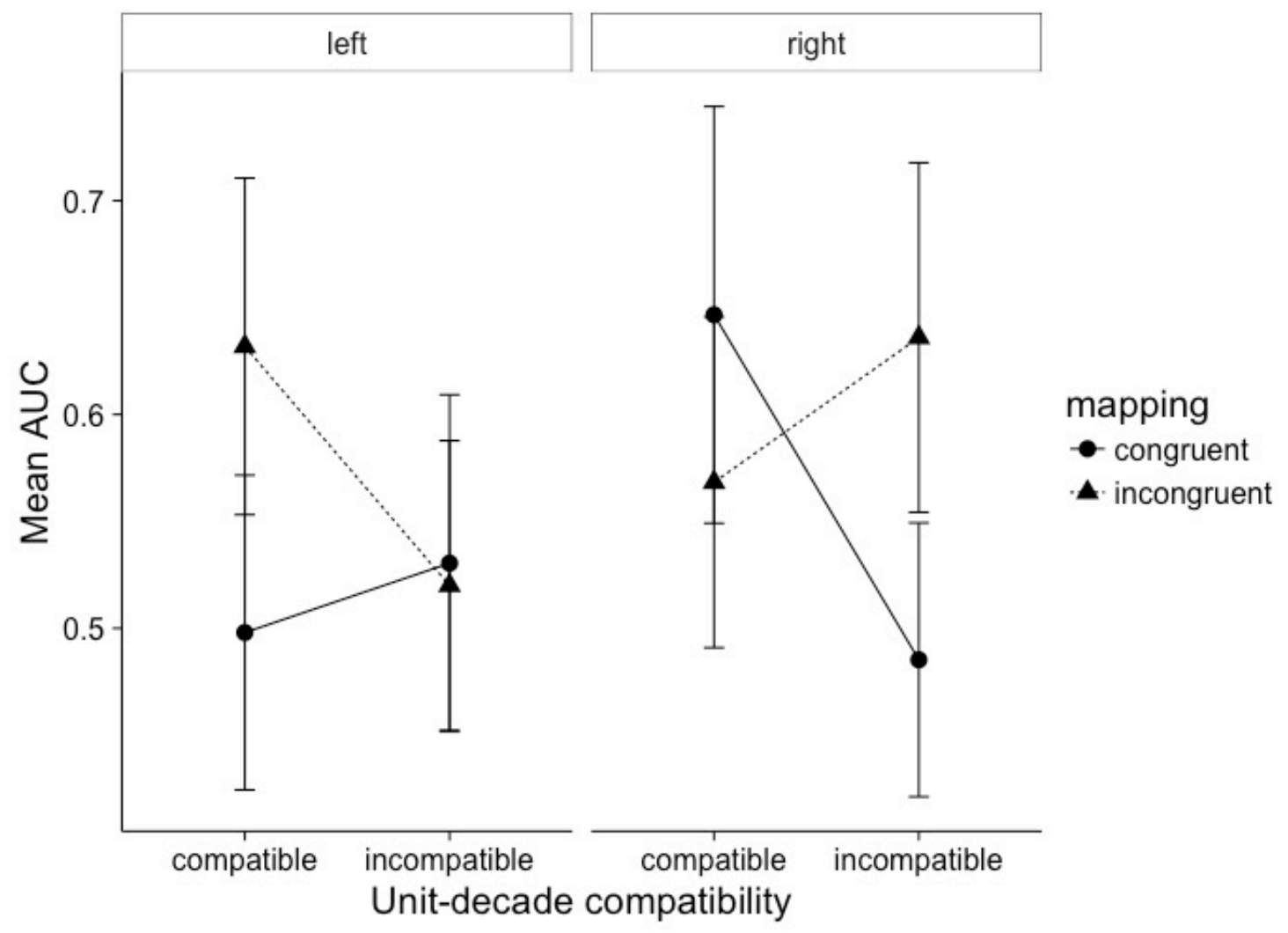

Figure 7. Mean AUC values as a function of unit-decade compatibility (compatible, incompatible), response side (left, right), and response mapping (congruent, incongruent). Error bars represent within-subject 95\% confidence intervals as recommended by Morey (201)8).

With regard to decomposed processing, we found that participants did exhibit certain response signatures that were indicative of their attending to the decades and units of twodigit numbers separately. Though many studies have previously shown this to be the case, the present study is one of a very small number of studies (Dotan \& Dehaene, 20113; Bloechle et al., (2015) to examine the response dynamics of such decomposed processing. Both Dotan and Dehaene (2013) and Bloechle et al. (2015) showed that the unit digit has a significant influence on manual responses. Specifically, Bloechle et al. (2015) found an overall leftward bias in manual pointing positions that was reduced for unit-decade incompatible numbers, where separate comparison of decades and units leads to opposite decisions. Bloechle et al. (2015) hypothesized that this was due to an embodied representation of two-digit number, where long term memory traces have decades linked spatially with leftward space and units with rightward space. We demonstrated a version of this bias in our mouse trajectories, with trajectories for unit decade incompatible targets tracing rightward of trajectories for compatible targets. Critically, though, we were able to reverse this bias when the response 
mapping was reversed from LEFT=SMALLER to LEFT=LARGER. That is, trajectories for unit decade incompatible targets traced to the left of the trajectories for compatible targets. Such behavior cannot be explained by an embodiment view of two-digit number representation (Bloechle et al., 2015), and instead supports a model where decision dynamics stem from competition among parallel and partially active responses (Freeman, Dale, \& Farmer, 20II). Of course, the present work is not sufficient to completely specify the architecture of such a model, so this will likely be an exciting future avenue for research.

One curious result in the present study is that RT effects were isolated to movement times; our manipulations had no effect on initiation times. This runs counter to the results of Ishihara et al. (2006), who found differences in initiation latency but no significant differences in movement times. Ishihara et al. (2006) concluded that any interference between number and spatial representation was purely limited to an early, motor-planning stage. However, other recent works indicate that the opposite may be true. Indeed, both Song and Nakayama (2008) and Faulkenberry et al. (2016) found differences only in reach curvature and/or movement times rather than initiation times. One explanation for these results is likely attributable to the distinct components of action control. Specifically, initiation latency is thought to reflect a response threshold adjustment process related to the inhibition of motor output, whereas movement time and curvature reflect an ongoing monitoring process representing the coactivation of competing responses throughout a trial (Erb, Moher, Sobel, \& Song, 2016). This is consistent with our interpretation of the results of the present study, where interference emerges later during execution of reaching movements independent of the timing of motor preparation and initiation (see also Ruitenberg et al., 2016; Strauss, Woodgate, Sami, \& Heinke, 2015, for a discussion on this topic). Future studies should attempt to elucidate the distinct mechanisms underlying motor control in these tasks; doing so will help us to further pinpoint the exact nature of these processing signatures.

While the present study provides an important step toward the description of response dynamics in two-digit number comparison, some inherent limitations persist. First, the design of our study did not allow us to rule out whether participants could be simply attending to the decade when making two-digit number decisions. As the aim of our study was to investigate the dynamics of processing for each type of representational signature, we simply did not design a strong test between holistic and decomposed processing. Also, though we were able to test between competing hypotheses for the response signatures that were indicative of each of two main types of number representation (holistic and decomposed), the design of the study did not allow us to explicitly measure the relative contribution of each type of processing. Indeed, it is currently unclear to what extent the formation of two-digit number representations are driven by holistic and decomposed processing. Certainly, the 
fact that we saw robust signatures of both holistic and decomposed processing likely rules out both a purely holistic model (e.g., Hinrichs et al., ए981; Dehaene et al., 1990) and a strictly decomposed model (e.g., Verguts \& De Moor, 2005). As such, the support that the current work lends to a hybrid model (Nuerk \& Willmes, 200.5) is purely by elimination, so future work should set out to explicitly test this model in light the predictions it might make about response dynamics.

Also, we were a bit puzzled to find an overall reversed unit decade compatibility effect, where incompatible numbers were compared slightly faster than compatible numbers. Usually, the pattern is reversed, with longer RTs for incompatible trials. One possible reason for this unique result stems from our chosen stimulus set. Although absolute distances from the comparison standard were matched fairly closely across all four distance and compatibility conditions (see Table 1), the distances were not matched well between the "less than 55" and "greater than 55" stimuli. For example, on compatible trials, the mean absolute distance for numbers less than 55 was 26.875, whereas the mean absolute distance for numbers greater than 55 was 17.125. On the other hand, mean absolute distance was well matched for incompatible trials (22.5 for numbers less than 55, 22.5625 for numbers larger than 55). This imperfect matching of stimuli between conditions likely contributed to our reversed compatibility effect, as distances were slightly larger overall for incompatible numbers compared to compatible numbers. Also, there were larger decade distances for our incompatible numbers. Both of these patterns would work against the usual compatibility effect, as comparisons with greater distances are usually facilitated (e.g., the numerical distance effect, Moyer \& Landauer, 1.967).

It is also possible that the design of the experimental apparatus may have impacted the patterns observed in our participants' movement trajectories. If the degree of curvature present in a reach movement strongly depended on the location of the responding hand, the fact that our participants all used a right-handed motion with the computer mouse should have resulted in asymmetrical effects of distance and unit-decade compatibility. Inspecting the degree of competition in participants' leftward and rightward responses, there are no apparent asymmetries when manipulating numerical distance (see Figures B and 四). For decomposed processing signatures, however, a significant three-way interaction between compatibility, response mapping, and response side (see Figures $\mathbf{6}$ and प) may reveal an ipsilateral effect, where distractors on the same side of space as the effector cause greater interference effects than distractors on the opposite side of space. For our study, the relative magnitudes of any interference effects that were generated by distractors could be slightly stronger for right side responses because our participants always used the right hand in their responses. Such a pattern of interference would be consistent with evidence showing that the Simon effect is influenced by handedness, with an advantage for the dominant 
hand when it is used to execute a response in the corresponding space (e.g., Arend, Weiss, Timpert, Fink, \& Henik, 2016; Rubichi \& Nicoleti, 20106). For instance, Rubichi and Nicoleti (2006) examined the relation between handedness and the size of the Simon effect in each visual hemifield. They found that the Simon effect was larger in the right visual hemifield in right-handers and in the left visual field in left-handers. While there are also studies that reported a reduction of the Simon effect in the field of the responding hand (Arend et al., 2016), they are all in agreement in showing that factors associated with the location of the effectors can affect spatial coding. On the whole, authors of these studies explained such findings by postulating close links between the processes of action planning and attention, with an attentional bias centered on the dominant hand. Interestingly, Bloechle et al. (2015) also found asymmetries in pointing responses as a function of hand, with different landing positions and reaction times for left-hand and right-hand responses. In sum, close links between the attentional and the motor systems may have generated some processing advantage when participants made ipsilateral reaches. With this is mind, however, we stress that our study was not designed to investigate the individual contribution of these factors. Future studies will need to examine how factors associated with handedness and location of response effectors will each affect measures of reach movement.

More broadly, the present work adds to the growing body of literature in numerical cognition that shows support for competitive processing in number tasks ranging among single digit number comparison (Faulkenberry, 2016; Santens et al., 201), numerical parity (Faulkenberry, 2014), fraction comparison (Faulkenberry et al., 2015), comparison of number pairs (Ganor-Stern \& Goldman, 2014), and physical size comparison (Faulkenberry et al., 2016). This work provides empirical data in support of recent computational models of number representation (Verguts et al., 2005; Gevers, Verguts, Reynvoet, Caessens, \& Fias, (2006) and showing that a purely embodied perspective is likely insufficient to account for the observed associations between symbolic number and space (Santens \& Gevers, 2008; Gevers et al., 2010). Further, the present study adds to a growing body of work on the dynamics of cognitive processing, representing diverse topics such as stereotype formation (Freeman \& Ambady, [200.9), voice processing (Sulpizio et al., 2015), language comprehension (Spivey et al., 2010.5; Incera \& McLellan, 2015), memory (Abney, McBride, Conte, \& Vinson, 2014; Papesh \& Goldinger, 2012), and face processing (Freeman \& Ambady, 2011; Hehman, Carpinella, Johnson, Leitner, \& Freeman, 2014).

In summary, the present study shows that in two-digit number comparison, adults show evidence of both holistic and decomposed processing. Though this is not the first time such response dynamics have been observed, our study did provide the first evidence that these response patterns are not due to a direct mapping between an embodied number representation and external space, but are rather likely the result of task specific demands 
that promote competition between parallel and partially active response options.

References

Abney, D. H., McBride, D. M., Conte, A. M., \& Vinson, D. W. (2014). Response dynamics in prospective memory. Psychonomic Bulletin \& Review, 22(4), 1020-1028. doi:11. 3758/s13423-014-0771-6

Arend, I., Weiss, P. H., Timpert, D. C., Fink, G. R., \& Henik, A. (2016). Spatial coding as a function of handedness and responding hand: Theoretical and methodological implications. PLOS ONE, 11(3), e0151979. doi:10.1371/journal.pone.0151979

Barsalou, L. W. (2008). Grounded cognition. Annual Review of Psychology, 59(1), 617-645. doi:10.1146/annurev.psych.59.103006.093639

Bloechle, J., Huber, S., \& Moeller, K. (2015). In touch with numbers: Embodied and situated effects in number magnitude comparison. Journal of Cognitive Psychology, 27(4), 478489. doi:10.1080/20445911.2014.1001760

Dehaene, S., Dupoux, E., \& Mehler, J. (1990). Is numerical comparison digital? Analogical and symbolic effects in two-digit number comparison. Journal of Experimental Psychology: Human Perception and Performance, 16 (3), 626-641. doi:10.1037/00961.52 .3 .16 .3 .626

Dotan, D. \& Dehaene, S. (2013). How do we convert a number into a finger trajectory? Cognition, 129(3), 512-529. doi:10.1016/j.cognition.2013.07.007

Erb, C. D., Moher, J., Sobel, D. M., \& Song, J.-H. (2016). Reach tracking reveals dissociable processes underlying cognitive control. Cognition, 152, 114-126. doi:10.1016/j. cognition.2016.03.015

Faulkenberry, T. J. (2014). Hand movements reflect competitive processing in numerical cognition. Canadian Journal of Experimental Psychology, 68(3), 147-151. doi:10.1037/ cep0000021

Faulkenberry, T. J. (2016). Testing a direct mapping versus competition account of response dynamics in number comparison. Journal of Cognitive Psychology. doi:110. 1080/20445911.2016.1191504

Faulkenberry, T. J., Cruise, A., Lavro, D., \& Shaki, S. (2016). Response trajectories capture the continuous dynamics of the size congruity effect. Acta Psychologica, 163, 114-123. doi:10.1016/j.actpsy.2015.11.010

Faulkenberry, T. J., Montgomery, S. A., \& Tennes, S.-A. N. (2015). Response trajectories reveal the temporal dynamics of fraction representations. Acta Psychologica, 159, 100107. doi:10.1016/j.actpsy.2015.05.013 
Faulkenberry, T. J. \& Rey, A. E. (2014). Extending the reach of mousetracking in numerical cognition: A comment on fischer and hartmann (2014). Frontiers in Psychology, 5. doi:10.3389/fpsyg.2014.01436

Faulkenberry, T. J. \& Tummolini, L. (2016). Commentary: Is there any influence of variations in context on object-affordance effects in schizophrenia? Perception of property and goals of action. Frontiers in Psychology, 7, 1915. doi:10.3389/fpsyg.2016.01915

Fischer, M. H. (2008). Finger counting habits modulate spatial-numerical associations. Cortex, 44 (4), 386-392. doi:10.1016/j.cortex.2007.08.004

Fischer, M. H. \& Hartmann, M. (2014). Pushing forward in embodied cognition: May we mouse the mathematical mind? Frontiers in Psychology, 5(1315). doi:10.3389/fpsyg. 20114.111315

Freeman, J. B. \& Ambady, N. (2009). Motions of the hand expose the partial and parallel activation of stereotypes. Psychological Science, 20(10), 1183-1188. doi:10.1111/j. $1467-9280.2009 .02422 . x$

Freeman, J. B. \& Ambady, N. (2010). MouseTracker: Software for studying real-time mental processing using a computer mouse-tracking method. Behavior Research Methods, 42(1), 226-241. doi:10.3758/BRM.42.1.226

Freeman, J. B. \& Ambady, N. (2011). Hand movements reveal the time-course of shape and pigmentation processing in face categorization. Psychonomic Bulletin 8 Review, 18(4), 705-712. doi:10.3758/s13423-011-0097-6

Freeman, J. B., Dale, R., \& Farmer, T. A. (2011). Hand in motion reveals mind in motion. Frontiers in Psychology, 2(59). doi:10.3389/fpsyg.2011.00059

Ganor-Stern, D. \& Goldman, R. (2014). Reaching towards an end: Numerical end and distance effects in motor movements. Journal of Cognitive Psychology, 27(4), 490498. doi:10.1080/20445911.2014.950662

Gevers, W., Santens, S., Dhooge, E., Chen, Q., den Bossche, L. V., Fias, W., \& Verguts, T. (2010). Verbal-spatial and visuospatial coding of numberspace interactions. Journal of Experimental Psychology: General, 139(1), 180-190. doi:10.1037/a0017688

Gevers, W., Verguts, T., Reynvoet, B., Caessens, B., \& Fias, W. (2006). Numbers and space: A computational model of the SNARC effect. Journal of Experimental Psychology: Human Perception and Performance, 32(1), 32-44. doi:10.1037/0096-1523.32.1.32

Haslbeck, J. M. B., Wood, G., \& Witte, M. (2015). Temporal dynamics of number-space interaction in line bisection: Comment on cleland and bull (2015). The Quarterly Journal of Experimental Psychology, 1-7. doi:10.1080/17470218.2015.1095773

Hehman, E., Carpinella, C. M., Johnson, K. L., Leitner, J. B., \& Freeman, J. B. (2014). Early processing of gendered facial cues predicts the electoral success of female politi- 
cians. Social Psychological and Personality Science, 5(7), 815-824. doi:10.1177/ $19485.506145: 34701$

Hinrichs, J. V., Yurko, D. S., \& Hu, J.-M. (1981). Two-digit number comparison: Use of place information. Journal of Experimental Psychology: Human Perception and Performance, 7(4), 890-901. doi:10.1037/0096-1523.7.4.890

Incera, S. \& McLellan, C. T. (2015). Mouse tracking reveals that bilinguals behave like experts. Bilingualism, 19(03), 610-620. doi:10.1017/s1366728915000218

Ishihara, M., Jacquin-Courtois, S., Flory, V., Salemme, R., Imanaka, K., \& Rossetti, Y. (2006). Interaction between space and number representations during motor preparation in manual aiming. Neuropsychologia, 44 (7), 1009-1016. doi:10.1016/j. neuropsychologia.2005.11.008

Jeffreys, H. (1961). The Theory of Probability (3rd ed.) Oxford, UK: Oxford University Press.

Lindemann, O., Abolafia, J. M., Girardi, G., \& Bekkering, H. (2007). Getting a grip on numbers: Numerical magnitude priming in object grasping. Journal of Experimental Psychology: Human Perception and Performance, 33(6), 1400-1409. doi:10.1037/ $00.96-1523.33 .6 .1400$

Lindley, D. V. (1957). A statistical paradox. Biometrika, 44(1-2), 187-192. doi:10.1093/ biomet/44.1-2.187

Marghetis, T., Núñez, R., \& Bergen, B. K. (2014). Doing arithmetic by hand: Hand movements during exact arithmetic reveal systematic, dynamic spatial processing. The Quarterly Journal of Experimental Psychology, 67(8), 1579-1596. doi:10.1080/ 17470218.2014 .897359

Masson, M. E. J. (2011). A tutorial on a practical Bayesian alternative to null-hypothesis significance testing. Behavior Research Methods, 43(3), 679-690. doi:10.3758/s13428(III)-(1)(14.9-5)

McCloskey, M. (1992). Cognitive mechanisms in numerical processing: Evidence from acquired dyscalculia. Cognition, 44(1-2), 107-157. doi:10.1016/0010-0277(92)90052-j

Moeller, K., Fischer, M. H., Nuerk, H.-C., \& Willmes, K. (2009). Sequential or parallel decomposed processing of two-digit numbers? Evidence from eye-tracking. The Quarterly Journal of Experimental Psychology, 62 (2), 323-334. doi:10.1080/17470210801946740

Morey, R. D. (2008). Confidence intervals from normalized data: A correction to cousineau (2005). Tutorial in Quantitative Methods for Psychology, 4(2), 61-64.

Moyer, R. S. \& Landauer, T. K. (1967). Time required for judgements of numerical inequality. Nature, 215, 1519-1520. doi:10.1038/2151519a0 
Nuerk, H.-C., Kaufmann, L., Zoppoth, S., \& Willmes, K. (2004). On the development of the mental number line: More, less, or never holistic with increasing age? Developmental Psychology, 40 (6), 1199-1211. doi:10.1037/0012-1649.40.6.1199

Nuerk, H.-C., Weger, U., \& Willmes, K. (2001). Decade breaks in the mental number line? Putting the tens and units back in different bins. Cognition, 82(1), B25-B33. doi:10. 1016/s0010-0277(01)00142-1

Nuerk, H.-C. \& Willmes, K. (2005). On the magnitude representations of two-digit numbers. Psychology Science, 47(1), 52-72.

Papesh, M. H. \& Goldinger, S. D. (2012). Memory in motion: Movement dynamics reveal memory strength. Psychonomic Bulletin \& Review, 19(5), 906-913. doi:10.3758/ S1:342:3-1||2-1128|-3

Proctor, R. W. \& Cho, Y. S. (2006). Polarity correspondence: A general principle for performance of speeded binary classification tasks. Psychological Bulletin, 132(3), 416-442. doi:10.1037/0033-2909.132.3.416

R Development Core Team. (2011). R: A language and environment for statistical computing. ISBN 3-900051-07-0. R Foundation for Statistical Computing. Vienna, Austria. Retrieved from http://www.R-project.org/

Restle, F. (1970). Speed of adding and comparing numbers. Journal of Experimental Psychology, 83(2, Pt.1), 274-278. doi:10.1037/h0028573

Reynvoet, B., Notebaert, K., \& Van den Bussche, E. (2011). The processing of two-digit numbers depends on task instructions. Zeitschrift für Psychologie, 219(1), 37-41. doi:10.1027/2151-2604/a000044

Rubichi, S. \& Nicoleti, R. (2006). The simon effect and handedness: Evidence for a dominanthand attentional bias in spatial coding. Perception \& Psychophysics, 68 (7), 1059-1069. doi:10.3758/bf03193709

Ruitenberg, M. F. L., Duthoo, W., Santens, P., Seidler, R. D., Notebaert, W., \& Abrahamse, E. L. (2016). Sequence learning in parkinson's disease: Focusing on action dynamics and the role of dopaminergic medication. Neuropsychologia, 93, 30-39. doi:10.1016/j. neuropsychologia.2016.09.027

Santens, S. \& Gevers, W. (2008, July). The SNARC effect does not imply a mental number line. Cognition, 108(1), 263-270. doi:10.1016/j.cognition.2008.01.002

Santens, S., Goossens, S., \& Verguts, T. (2011). Distance in motion: Response trajectories reveal the dynamics of number comparison. PloS One, 6(9), e25429. doi:10.1371/ journal.pone.0025429

Shaki, S., Fischer, M. H., \& Petrusic, W. H. (2009). Reading habits for both words and numbers contribute to the SNARC effect. Psychonomic Bulletin \& Review, 16(2), 328-331. doi:10.3758/pbr.16.2.328 
Song, J.-H. \& Nakayama, K. (2008). Numeric comparison in a visually-guided manual reaching task. Cognition, 106(2), 994-1003. doi:10.1016/j.cognition.2007.03.014

Spivey, M. J. (2007). The continuity of mind. New York: Oxford University Press.

Spivey, M. J., Grosjean, M., \& Knoblich, G. (2005). Continuous attraction toward phonological competitors. Proceedings of the National Academy of Sciences, 102(29), 1039310398. doi:10.1073/pnas.0503903102

Strauss, S., Woodgate, P. J., Sami, S. A., \& Heinke, D. (2015). Choice reaching with a LEGO arm robot (CoRLEGO): The motor system guides visual attention to movementrelevant information. Neural Networks, 72, 3-12. doi:10.1016/j.neunet.2015.10.005

Sulpizio, S., Fasoli, F., Maass, A., Paladino, M. P., Vespignani, F., Eyssel, F., \& Bentler, D. (2015). The sound of voice: Voice-based categorization of speakers' sexual orientation within and across languages. PLOS ONE, 10(7), e0128882. doi:10.1371/journal.pone. 0128882

Verguts, T. \& De Moor, W. (2005). Two-digit comparison: Decomposed, holistic, or hybrid? Experimental Psychology, 52(3), 195-200. doi:10.1027/1618-3169.52.3.195

Verguts, T., Fias, W., \& Stevens, M. (2005). A model of exact small-number representation. Psychonomic Bulletin \& Review, 12(1), 66-80. doi:10.3758/bf03196349

Wagenmakers, E.-J. (2007). A practical solution to the pervasive problems of $p$ values. Psychonomic Bulletin \& Review, 14(5), 779-804. doi:10.3758/bf03194105

Wood, G., Nuerk, H. C., Freitas, P., Freitas, G., \& Willmes, K. (2006). What do semiilliterate adults know about 2-digit arabic numbers? Cortex, 42(1), 48-56. doi:11. 1016/s0010-9452(08)70321-6 